\title{
Betatrophin provides a new insight into diabetes treatment and lipid metabolism (Review)
}

\author{
JIN-ZHOU ZHU, CHAO-HUI YU and YOU-MING LI \\ Department of Gastroenterology and Hepatology, The First Affiliated Hospital, \\ College of Medicine, Zhejiang University, Hangzhou, Zhejiang 310003, P.R. China \\ Received March 21, 2014; Accepted May 16, 2014
}

DOI: $10.3892 /$ br.2014.284

\begin{abstract}
Replenishing the insulin-producing $\beta$-cell mass is considered to be a potential cure for diabetes. A recent study identified a secreted protein, known as betatrophin, which potently induces pancreatic $\beta$-cell proliferation. Notably, a number of studies reportedly identified betatrophin, which is also known as lipasin, atypical angiopoietin-like 8 and refeeding-induced fat and liver protein, and considered to be a novel regulator in lipid metabolism according to the studies. The identification of betatrophin was considered to create novel opportunities for potential diabetes therapy. In the present study, the current knowledge of betatrophin is reviewed, with regards to its character and function in lipid homeostasis and pancreatic $\beta$-cell proliferation.
\end{abstract}

\section{Contents}

1. Introduction

2. Refeeding induced fat and liver

3. Angiopoietin-like 8

4. Lipasin, lipoprotein lipase inhibition

5. Betatrophin, a $\beta$-cell booster

6. Conclusion

\section{Introduction}

According to the data from the International Diabetes Federation, the global prevalence of diabetes in 2011 was 366 million. The number is estimated to reach 552 million with a $7.7 \%$ comparative prevalence by 2030 (1).

Correspondence to: Dr You-Ming Li, Department of Gastroenterology and Hepatology, The First Affiliated Hospital, College of Medicine, Zhejiang University, 79 Qingchun Lu, Hangzhou, Zhejiang 310003, P.R. China

E-mail: zlym@zju.edu.cn

Key words: diabetes, insulin resistance, betatrophin, $\beta$ cell
The main occurrence of diabetes is type II diabetes, which is characterized by a series of metabolic disorders that consist of impaired $\beta$-cell function, insulin resistance (IR) and exhaustion, dedifferentiation and loss of functional $\beta$-cell mass (2-4). By contrast, type I diabetes appears to result from an autoimmune attack on pancreatic $\beta$ cells, which consequently leads to a $\beta$-cell deficiency (5). Currently, there is no pharmacological treatment or insulin injection available that can substitute for the endogenous $\beta$-cell function entirely in the prevention of hyperglycemia and the resulting complications.

To the best of our knowledge, the pancreatic $\beta$ cell is a perfect sensor for blood glucose levels and secretes an accurate amount of insulin to regulate glucose and energy homeostasis. A therapy that restores the insulin-producing pancreatic $\beta$-cell mass is likely to benefit all diabetics, even though the primary causes of type I and type II diabetes are different. Restoration of the functional $\beta$-cell mass is therefore expected to be a key aim of diabetes therapy (6).

Based on this theory, numerous studies (?) have been reported in the last few decades that have aimed to identify novel factors that are involved in the regulation of $\beta$-cell mass and may provide novel potential therapeutic strategies. Gut-derived incretins, muscle-derived myokines, macrophage-derived cytokines and adipocyte-derived adipokines were expected to develop regenerative $\beta$-cell therapy (7). However, these factors contribute to enhancing the $\beta$-cell mass and function, although the clinical practice has been restricted due to limited specificity and uncertain effects (8).

In 2010, Genentech and Lexicon Pharmaceutical Collaboration created a library of knock-out murine models that targeted the genes encoding secreted and membrane proteins, providing 472 murine mutant lines that were subjected to a systematic phenotypic screen (9). In the mice knock-out library, the deletion of the Gm6484 gene was found to significantly reduce serum triglyceride (TG) levels, compared to wild-type mice. In another study concerning genome screening for common variants associated with human lipid compounds, the variations of C19orf80, the human homologue of Gm6484, were associated with plasma lipid levels (10).

Yi et al (11) also identified betatrophin, a novel hormone that is increased in mice that exhibit insulin receptor antagonist-induced IR, and also induces a notable dose-dependent increase in pancreatic $\beta$-cell proliferation. Identification of betatrophin exerting control on $\beta$-cell mass and replication, 
is considered to create novel opportunities for potential diabetes therapy. Notably, other studies have also recently reported the identification and function of betatrophin, which was designated as lipasin $(12,13)$, atypical angiopoeitin-like 8 (ANGPTL8) (14) and refeeding-induced fat and liver protein (RIFL) (15). In those studies, this $\beta$-cell booster was recognized as a nutritionally-regulated factor that controls serum lipid metabolism.

In the present study, the knowledge pertaining to betatrophin is reviewed with regards to its character and function in lipid homeostasis and pancreatic $\beta$-cell proliferation.

\section{Refeeding induced fat and liver}

In 2012, Ren et al (15) utilized the Lexicon-Genentech mice gene knock-out library and generated 3T3-L1 cells in vitro adipogenesis. The study reported the Gm6484 gene as RIFL due to the high induction of RIFL during adipogenesis in the primary cultures of rodent and human adipocytes and in 3T3-L1 cells. Knockdown of the RIFL gene leads to reduced adipogenesis, which presented a reduced TG content.

In mice, RIFL mRNA was highly expressed in white and brown adipose tissue (WAT and BAT, respectively) and the liver. In particular, the WAT content was detected to undergo a significant increase in ob/ob mice, which is a model of genetic obesity due to the absence of leptin. Similarly in humans, the RIFL expression was enriched in liver and fat tissues. Additionally, in the 3T3-L1 adipocytes, the expression level of RIFL was induced by insulin in a time- and dose-dependent manner. Numerous agents that stimulate lipolysis have been shown to inhibit the expression of the RIFL gene.

These data showed several aspects of RIFL in adipocyte regulation and suggested that RIFL is a novel regulator of lipid metabolism.

\section{Angiopoietin-like 8}

Angiopoietin-like proteins (ANGPTLs) have main roles in lipid metabolism and trafficking (16). ANGPTL3, a gene located in an intron of DOCK7, has been shown to be involved in lipid homeostasis.

Quagliarini et al (14) reported that the Gm6484 gene is located in the corresponding intron of DOCK6 and the resulting product, known as ANGPTL8, is a paralog of ANGPTL3. As demonstrated previously, ANGPTL8 is largely expressed in mice liver, WAT, BAT and adrenal glands, whereas it is expressed primarily in the liver, adipose tissue and plasma of humans. ANGPTL8 was markedly upregulated by feeding and suppressed by fasting in mice and humans (13-15). Additionally, a variant in this family member (R59W, rs2278426) has been demonstrated to be associated with a decreased plasma low- and high-density lipoprotein cholesterol content in three populations (14).

The ANGPTL protein family includes 7 members (ANGPTL1 to 7). Similar to angiopoietins, all ANGPTLs have one or two coiled-coil domains and a fibrinogen-like domain (16-19). Previous studies have demonstrated that two closely associated members, ANGPTL3 and ANGPTL4, have essential roles in lipid and lipoprotein trafficking and metabolism (20-23). In the previous studies, the deletion of the ANGPTL3 gene was associated with significantly reduced plasma levels of TG and cholesterol in mice and humans $(20,22)$. Similarly, mice lacking ANGPTL4 also present with reduced levels of blood TG and cholesterol. Furthermore, sequence variations of ANGPTL4 have been confirmed to be associated with lower TG levels in humans $(22,23)$.

With regards to the similarities, ANGPTL8 shares homolog with the N-terminal region of ANGPTL3 and ANGPTL4, which facilitates lipasin, lipoprotein lipase (LPL) binding, and the three genes contribute to plasma TG regulation (14). However, in terms of differences, it should be noted that there are several distinct traits between ANGPTL8 and other members of the ANGPTL family $(13,24,25)$. As aforementioned, all ANGPTLs harbor fibrinogen-like domains and coiled-coil domains that do not exist in ANGPTL8. In addition, all ANGPTLs have various glycosylation sites and amino acids to establish disulphide bonds, whereas these are not present in ANGPTL8. These characteristics suggest that ANGPTL8 is a novel, but atypical member, in the ANGPTL protein family.

To assess the functional association between the two ANGPTL family members, Quagliarini et al (14) expressed ANGPTL3 at various levels in combination with or without ANGPTL8 in the livers of mice. As was suggested, ANGPTL8 interacted with ANGPTL3 and facilitated its cleavage. Consistent with this result, adenovirus-mediated overexpression of ANGPTL8 resulted in higher serum TG, whereas this result was reversed in ANGPTL $3^{-/}$mice. Additionally, ANGPTL8 expression was induced by food intake in a sterol-regulatory element-binding protein 1 independent manner. These data suggest that ANGPTL8, a paralog of ANGPTL3, regulates TG and fatty acid metabolism in response to food intake by controlling activation of its progenitor, possibly ANGPTL3.

Recently, the characteristics and function of ANGPTL8 were reported (26). The primary aim of the study was to investigate the effect of ANGPTL8 on lipid and glucose metabolism by using the ANGPTL8 gene knock-out mice. The results indicate that ANGPTL8 plays a pivotal role in the transition of metabolic status between fasting and refeeding. Furthermore, glucose and insulin-tolerance testing did not reveal significant changes in glucose homeostasis in mice consuming either a chow-fed or high-fat diet (HFD). Finally, immunoblot analysis revealed that ANGPTL8 is not required for ANGPTL3 cleavage.

\section{Lipasin, lipoprotein lipase inhibition}

Another study by Zhang (13), identified Gm6484 as a nutritional regulation gene by performing RNA sequencing on the mRNA of liver and fat tissues in mice that were exposed to various nutritional situations. It is widely accepted that 'in' denotes a circulating factor. Due to the inhibition of LPL activity, the gene was termed lipasin.

The study showed that human lipasin is liver-specific, while it is abundantly expressed in the liver, BAT and WAT of mice (13). HFD treatment markedly increased lipasin expression in the liver, whereas 24-h fasting markedly reduced it in fat and 48-h fasting reduced it in liver. In addition, adenoviruses induced the overexpression of lipasin in mice, which increased the serum TG levels. Recombinant lipasin at high 
concentrations inhibits LPL activity in vitro. Based on these results, it is considered that lipasin is a liver-enriched nutritional regulator that inhibits LPL and reduces TG clearance, thereby increasing the serum TG content.

Similarly, Fu et al (12) published another study regarding lipasin in the subsequent year. Considering the high expression in BAT, the thermoregulated and nutritionally-regulated functions of lipasin was investigated in BAT. The results showed that the lipasin expression levels markedly increased following exposure to a cold environment $\left(4^{\circ} \mathrm{C}\right.$ for $\left.4 \mathrm{~h}\right)$, while the levels of ANGPTL4 and ANGPTL2 were reduced. Furthermore, fasting evidently decreased lipasin expression in BAT, but increased ANGPTL4 expression. HFD treatment increases lipasin expression but reduces ANGPTL2.

The data indicated that the definitive physiological roles for ANGPTL family members involved the regulation of thermogenesis and energy homeostasis. Compared to ANGPLT4, lipasin in BAT presents an opposite role of thermoregulation and nutritional regulation. Similar to the aforementioned studies, these results supported that lipasin is a novel, but atypical, member of the ANGPTL protein family.

The studies that initially reported the identification and function of betatrophin (13-15) also revealved that the Gm6484/C19orf80 gene is a liver- and fat-enriched gene and provided a profile of the gene product in lipid metabolism, which demonstrated involvement in the regulation of the plasma TG level. However, there are several differences between the studies as well.

The studies differ with regards to the expression pattern among tissues. In mice, the study by Zhang (13) demonstrated that the gene mainly expresses in the liver and BAT rather than WAT. By contrast, Ren et al (15) and Quagliarini et al (14) suggested comparable expression levels in liver, BAT and WAT, as well as the adrenal gland. In humans, Zhang (13) showed that in addition to a low expression in WAT, the gene is principally expressed in the liver, whereas Quagliarini et al (14) indicated that there is considerable expression in WAT and in the brain. Due to the vulnerability of Gm6484/C19orf80 to the nutritional status, the different opinions on the expression pattern of the gene may attribute to various feeding regimens in the previous studies.

In terms of lipid metabolism, Quagliarini et al (14) suggested that the gene product increases lipolysis, owing to the assistance in ANGPTL3 cleavage. By contrast, Ren et al (15) showed that it is lipogenic. However, Zhang (13) supported the hypothesis that Gm6484/C19orf80 reduces TG clearance via LPL inhibition, thereby increasing the serum TG content. These apparently opposite characteristics of lipid metabolism require confirmation by further experiments.

\section{Betatrophin, a $\beta$-cell booster}

A previous study (14) provided a potential mechanism for LPL inhibition or an effect on serum TG level. However, no previous studies have reported the effects of the Gm6484/C19orf80 gene on pancreatic $\beta$ cell or diabetes.

A number of murine models that developed severe IR have been previously reported to undergo a clear increase in pancreatic mass. The increment in pancreatic mass observed in the
IR status reignited an exploration for the potential factors that control $\beta$-cell proliferation.

Michael et al (7) and Flier et al (27) reported a series of experiments that strongly supported that the factors that were secreted by the liver and released into the circulation promoted islet cell growth. Using liver-specific insulin receptor knock-out (LIRKO) mice, Flier et al (27) reported the induction of a factor that contributes to the crosstalk between liver and pancreatic islets that trigger $\beta$-cell proliferation. Subsequent to this, other studies have supported the existence of circulating factors that may induce $\beta$-cell proliferation $(8,28)$.

The study by Yi et al (11) reported the identification of an unknown factor that induces compensatory $\beta$-cell proliferation in a novel pharmacological marine model of IR. In this study, S961, a 43 amino acid inhibitory peptide that has a high affinity and selectivity for the insulin receptor, was infused into mice to induce an IR status. The results suggested that the hormone, betatrophin, enhances insulin production through induction of $\beta$-cell proliferation, thereby contributing to metabolism. Notably, betatrophin transgenic overexpression in liver resulted in a specific, rapid and robust increase of $\beta$-cell proliferation and an improved glucose tolerance in young adult mice. Additionally, the study revealed that betatrophin, a secreted protein of 198 amino acids, is highly conserved in mammalian species. In humans, betatrophin is mainly expressed in the liver. As shown in the study (11), betatrophin underwent upregulated expression in the liver and fat in a series of mice models that exhibited $\beta$-cell proliferation, including IR, pregnancy and leptin deficiency.

However, the study did not demonstrate the correlation between the circulating contents of betatrophin and the increment of pancreatic islet mass. Additionally, the study assessed a novel model of IR, developed by S961 that is an insulin-receptor antagonist, rather than traditional models, such as diet-induced obesity $(7,29,30)$. However, whether betatrophin undergoes similar changes in other genetic manipulations that provoke $\beta$-cell replication, including LIRKO and MEK1 mutations (7,31), remains to be investigated.

Subsequently, the administration of S961 was employed to determine its effect on human $\beta$ cells (32). The IR status developed by S961 was confirmed to cause a clear increase of $\beta$-cell replication in mice. In particular, this consequence occurred, regardless of whether the $\beta$ cells were in their native environment, such as the pancreas or in the ectopic location, or the kidney capsule in the study. By contrast, the human pancreatic islets transplanted under the kidney failed to respond. These results question the suitability of betatrophin in therapeutic approaches for human diabetes.

A recent cross-sectional study (33) evaluated the betatrophin concentrations by ELISA in the plasma of patients with type I diabetes and age-matched healthy controls. Although betatrophin was increased in the plasma of patients with type I diabetes compared to controls, the plasma contents did not correlate with a series of metabolic parameters. Notably, among the healthy controls the plasma betatrophin levels increased with age. This indicates that betatrophin-induced $\beta$-cell proliferation may be a beneficial physiological mechanism and that increased betatrophin contents in patients with type I diabetes may be explained by IR. However, the presence of this mechanism remains to be determined. 


\section{Conclusion}

Recently, the discovery of betatrophin has raised a number of questions. The following is a list of specific principal issues that require clarification in the future.

i) Establishing the mechanism behind how betatrophin induces this potent response in pancreatic $\beta$ cells is required. Identification of the betatrophin receptor and other potential co-factors or signaling pathways may benefit in elucidating how the liver and fat interact with the pancreas to regulate the $\beta$-cell mass (34).

ii) The role of betatrophin in lipid regulation. First, further studies are required to determine how betatrophin interacts with the other members of the ANGPTL protein family (35). Second, with regards to LPL having a pivotal role in the uptake process of fatty acids derived from TG, betatrophin, also known as lipasin, inhibits LPL activity and is emerging as an essential lipid regulator and a potentially therapeutic target (12). Third, exploring the mutual influence between betatrophin and lipid homeostasis in the pathological development of diabetes may be useful.

iii) Age and betatrophin-induced $\beta$-cell regeneration. As indicated in the cross-sectional study (33), plasma betatrophin concentrations in the younger patients were higher than the older individuals among the healthy controls. In addition, it has been demonstrated that both mice and humans undergo a decline in the $\beta$-cell replication rate in correlation with an increase in age $(36,37)$. Thus, it remains to be investigated whether the effect of betatrophin on $\beta$-cell mass is apparent in aged mice or mice with $\beta$ cell of a limited regenerative ability or dedifferentiation.

iv) Association with irisin. A novel myokine, known as irisin, was identified by Bostrom et al (38). Irisin enhances the total energy expenditure, prolongs life expectancy, reduces body weight and protects against diet-induced IR (39). A recent study by Zhang et al (40), reported that irisin increased the expression of betatrophin in primary rat adipocytes and 3T3-L1-derived adipocytes. The pathway between irisin and betatrophin may be involved in the pathological progression of IR, which would provide a novel insight for diabetes therapy and obesity (41).

v) Potential side-effects accompanied by betatrophin treatment. The beneficial regenerative effects on $\beta$-cell replication, potential adverse effects, on account of dyslipidemia or other metabolism disorders, require further investigation to demonstrate that betatrophin specifically acts on $\beta$ cells and does not influence other cell or organs in the mammalian body.

\section{References}

1. Lam DW and LeRoith D: The worldwide diabetes epidemic. Curr Opin Endocrinol Diabetes Obes 19: 93-96, 2012.

2. Scheen AJ and Lefèbvre PJ: Insulin action in man. Diabetes Metab 22: 105-110, 1996.

3. Talchai C, Xuan S, Lin HV, Sussel L and Accili D: Pancreatic $\beta$ cell dedifferentiation as a mechanism of diabetic $\beta$ cell failure. Cell 150: 1223-1234, 2012.

4. Teodoro JS, Gomes AP, Varela AT, Duarte FV, Rolo AP and Palmeira CM: Uncovering the beginning of diabetes: the cellular redox status and oxidative stress as starting players in hyperglycemic damage. Mol Cell Biochem 376: 103-110, 2013.

5. Kabelitz D, Geissler EK, Soria B, Schroeder IS, Fändrich F and Chatenoud L: Toward cell-based therapy of type I diabetes. Trends Immunol 29: 68-74, 2008.
6. Bonner-Weir S and Weir GC: New sources of pancreatic beta-cells. Nat Biotechnol 23: 857-861, 2005.

7. Michael MD, Kulkarni RN, Postic C, et al: Loss of insulin signaling in hepatocytes leads to severe insulin resistance and progressive hepatic dysfunction. Mol Cell 6: 87-97, 2000.

8. El Ouaamari A, Kawamori D, Dirice E, et al: Liver-derived systemic factors drive $\beta$ cell hyperplasia in insulin-resistant states. Cell Rep 3: 401-410, 2013.

9. Tang T, Li L, Tang J, et al: A mouse knockout library for secreted and transmembrane proteins. Nat Biotechnol 28: 749-755, 2010.

10. Teslovich TM, Musunuru K, Smith AV, et al: Biological, clinical and population relevance of 95 loci for blood lipids. Nature 466: 707-713, 2010.

11. Yi P, Park JS and Melton DA: Betatrophin: a hormone that controls pancreatic $\beta$ cell proliferation. Cell 153: 747-758, 2013.

12. Fu Z, Yao F, Abou-Samra AB and Zhang R: Lipasin, thermoregulated in brown fat, is a novel but atypical member of the angiopoietin-like protein family. Biochem Biophys Res Commun 430: 1126-1131, 2013

13. Zhang R: Lipasin, a novel nutritionally-regulated liver-enriched factor that regulates serum triglyceride levels. Biochem Biophys Res Commun 424: 786-792, 2012.

14. Quagliarini F, Wang Y, Kozlitina J, et al: Atypical angiopoietin-like protein that regulates ANGPTL3. Proc Natl Acad Sci USA 109: 19751-19756, 2012.

15. Ren G, Kim JY and Smas CM: Identification of RIFL, a novel adipocyte-enriched insulin target gene with a role in lipid metabolism. Am J Physiol Endocrinol Metab 303: E334-E351, 2012.

16. Mattijssen F and Kersten S: Regulation of triglyceride metabolism by Angiopoietin-like proteins. Biochim Biophys Acta 1821: 782-789, 2012.

17. Oike Y and Tabata M: Angiopoietin-like proteins - potential therapeutic targets for metabolic syndrome and cardiovascular disease. Circ J 73: 2192-2197, 2009.

18. Li C: Genetics and regulation of angiopoietin-like proteins 3 and 4. Curr Opin Lipidol 17: 152-156, 2006.

19. Miida T and Hirayama S: Impacts of angiopoietin-like proteins on lipoprotein metabolism and cardiovascular events. Curr Opin Lipidol 21: 70-75, 2010.

20. Musunuru K, Pirruccello JP, Do R, et al: Exome sequencing, ANGPTL3 mutations and familial combined hypolipidemia. N Engl J Med 363: 2220-2227, 2010.

21. Romeo S, Yin W, Kozlitina J, et al: Rare loss-of-function mutations in ANGPTL family members contribute to plasma triglyceride levels in humans. J Clin Invest 119: 70-79, 2009.

22. Koishi R, Ando Y, Ono M, et al: Angptl3 regulates lipid metabolism in mice. Nat Genet 30: 151-157, 2002.

23. Romeo S, Pennacchio LA, Fu Y, et al: Population-based resequencing of ANGPTL4 uncovers variations that reduce triglycerides and increase HDL. Nat Genet 39: 513-516, 2007.

24. Inukai K, Nakashima Y, Watanabe M, et al: ANGPTL3 is increased in both insulin-deficient and -resistant diabetic states. Biochem Biophys Res Commun 317: 1075-1079, 2004.

25. Yoon JC, Chickering TW, Rosen ED, et al: Peroxisome proliferator-activated receptor gamma target gene encoding a novel angiopoietin-related protein associated with adipose differentiation. Mol Cell Biol 20: 5343-5349, 2000.

26. Wang Y, Quagliarini F, Gusarova V, et al: Mice lacking ANGPTL8 (Betatrophin) manifest disrupted triglyceride metabolism without impaired glucose homeostasis. Proc Natl Acad Sci USA 110: 16109-16114, 2013.

27. Flier SN, Kulkarni RN and Kahn CR: Evidence for a circulating islet cell growth factor in insulin-resistant states. Proc Natl Acad Sci USA 98: 7475-7480, 2001.

28. Kulkarni RN, Mizrachi EB, Ocana AG and Stewart AF: Human $\beta$-cell proliferation and intracellular signaling: driving in the dark without a road map. Diabetes 61: 2205-2213, 2012.

29. Kinsey-Jones JS and Murphy KG: Current models and strategies in the development of antiobesity drugs. Ann NY Acad Sci 1245: 3-6, 2011.

30. Aydin S, Kuloglu T, Aydin S, et al: Expression of adropin in rat brain, cerebellum, kidneys, heart, liver and pancreas in streptozotocin-induced diabetes. Mol Cell Biochem 380: 73-81, 2013.

31. Imai J, Katagiri H, Yamada T, et al: Regulation of pancreatic $\beta$ cell mass by neuronal signals from the liver. Science 322: 1250-1254, 2008.

32. Jiao Y, Le Lay J, Yu M, Naji A and Kaestner KH: Elevated mouse hepatic betatrophin expression does not increase human $\beta$-cell replication in the transplant setting. Diabetes 63: 1283-1288, 2014. 
33. Espes D, Lau J and Carlsson PO: Increased circulating levels of betatrophin in individuals with long-standing type 1 diabetes Diabetologia 57: 50-53, 2014.

34. Raghow R: Betatrophin: a liver-derived hormone for the pancreatic $\beta$-cell proliferation. World J Diabetes 4: 234-237, 2013.

35. Zhang R and Abou-Samra AB: Emerging roles of lipasin as a critical lipid regulator. Biochem Biophys Res Commun 432: 401-405, 2013.

36. Smukler SR, Arntfield ME, Razavi R, et al: The adult mouse and human pancreas contain rare multipotent stem cells that express insulin. Cell Stem Cell 8: 281-293, 2011.

37. Kushner JA: The role of aging upon $\beta$ cell turnover. J Clin Invest 123: 990-995, 2013.
38. Boström P, Wu J, Jedrychowski MP, et al: A PGC1- $\alpha$-dependent myokine that drives brown-fat-like development of white fat and thermogenesis. Nature 481: 463-468, 2012.

39. Novelle MG, Contreras C, Romero-Picó A, López M and Diéguez C: Irisin, two years later. Int J Endocrinol 2013: 746281, 2013.

40. Zhang Y, Li R, Meng Y, et al: Irisin stimulates browning of white adipocytes through mitogen-activated protein kinase p38 MAP kinase and ERK MAP kinase signaling. Diabetes 63: 514-525, 2014.

41. Sanchis-Gomar F and Perez-Quilis C: The p38-PGC-1 $\alpha$-irisinbetatrophin axis: exploring new pathways in insulin resistance. Adipocyte 3: 67-68, 2014 